\title{
Aprender matemáticas con ordenadores
}

\section{Eduardo Martí}
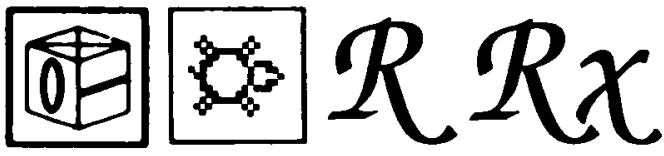

Utilizar el ordenador en la escuela no es una garantía de éxito didáctico si no se cumplen algunos requisitos, pero constituye una ayuda no suficientemente explorada en la mayoría de las materias. En el caso de las matemáticas, el ordenador permite diseñar actividades altamente motivadoras para el niño, a la vez que facilitar la integración de diferentes tipos de códigos, objetivos ambos difíciles de conseguir en los formatos de papel y lápiz habituales en las didácticas tradicionales. El autor de este artículo pasa revista a una serie de micromundos informáticos que permiten al profesor y al alumno librarse de tareas rutinarias y dedicarse a la exploración de las posibilidades del lenguaje matemático, a la verdadera tarea de aprender.

Las matemáticas constituyen un medio simbólico único (1). Al igual que otros medios simbólicos como pueden ser el lenguaje (en sus modalidades oral y escrita), los gestos, las imágenes (estáticas - dibujos, fotografías, gráficos- o dinámicas - cine, vídeo-) o la informática, las matemáticas ofrecen un doble aspecto. Por un lado, los símbolos matemáticos y las reglas y operaciones que rigen su sistema de notación pueden representar conceptos y procesos de una realidad no matemática: podemos, por ejemplo, operar utilizando símbolos numéricos que se aplican a un grupo de objetos en vez de operar directamente sobre éstos. Pero al mismo tiempo, las propiedades internas del medio matemático (distintas de las del lenguaje, de los gestos o de las imágenes) hacen que su utilización modifique la representación que tenemos de la realidad (al simbolizar un conjunto de objetos por un número abstraigo una de las propiedades de este conjunto dejando de lado otras propiedades) facilitando de manera radical el conocimiento que es posible tener de ella (qué difícil sería hacer una división con números elevados sin una formulación matemática y qué complicado buscar la solución de un problema que combina varias operaciones sin la simplifcación y la notación algebráica).

No es pues indiferente utilizar uno u otro medio para el conocimiento de la realidad. Este conocimiento puede ser más o menos adecuado a la finalidad perseguida (sería tan absurdo utilizar únicamente una descripción verbal para 
explicar a alguien la situación exacta de la ciudad de Bruselas pudiendo manejar un mapa, como utilizar únicamente una representación icónica para simbolizar los datos de una transacción comercial pudiendo calcularla artiméticamente). La utilización de uno $\mathrm{u}$ otro medio afecta pues el tipo de conocimiento que tenemos de la realidad y la elección adecuada de un medio puede liberar una parte del trabajo mental (memoria, atención) que sería necesario emplear si utilizásemos otro medio menos adecuado.

No creamos sin embargo que los diferentes medios simbólicos constituyen compartimentos estancos. Las relaciones entre diferentes códigos son importantísimas; la notación matemática presupone, por ejemplo, ciertas habilidades básicas de la lengua escrita, el lenguaje de los gestos, comparte ciertas características espaciales con el medio icónico y el lenguaje oral, comparte a su vez ciertas propiedades de orden temporal con el medio gestual. No olvidemos tampoco que una de las características de la cognición es precisamente la utilización complementaria de diferentes medios simbólicos y que una de las demandas cognitivas frencuentes en muchas de las tareas que debemos resolver es la traducción de un código simbólico a otro.

De estas consideraciones se pueden extraer tres postulados básicos:

1. El conocimiento de la realidad está mediatizado

2. Cada medio conduce a una representación distinta de la realidad

3. Aunque un medio pueda ser más apropiado que otro para el conocimiento de cierto aspecto de la realidad, conocer supone la utilización simultánea de medios distintos.

Los ordenadores crean, por sus particularidades de funcionamiento, un nuevo medio (el medio informático) cuya utilización puede aportar un cambio importante en la cognición, y por tanto en el aprendizaje y la enseñanza de las diferentes disciplinas (Martí, 1990; Olson, 1985; Pea, 1985).

Una de las cualidades de los ordenadores es su multifuncionalidad. Gracias a sus posibilidades de almacenar y manipular símbolos de manera dinámica (que serán presentados al usuario en forma de notaciones numéricas, escritura, sonido, imágenes, etc.), los ordenadores pueden ser utilizados para alcanzar una gama extensa de objetivos: cálculo, procesamiento de texto, análisis de imágenes, comunicación, almacenamiento de informaciones, etc. A pesar de esta diversidad de funciones, el medio informático presenta una serie de características que pueden transformar la experiencia matemática de los alumnos.

Pero tenemos que ser prudentes a la hora de juzgar el impacto que los ordenadores tienen sobre el aprendizaje de las matemáticas pues son innumerables los factores que pueden incidir sobre el proceso de enseñanza-aprendizaje con ordenadores en un contexto escolar. Nuestro análisis se centra aquí en el medio informático y sus caracerísticas pero es ilusorio pensar que, por sí solos, los ordenadores pueden modificar de manera positiva (o negativa) el aprendizaje de las matemáticas. Sería ésta una visión totalmente «tecnocéntrica» e ingenua. La repercusión que la interacción con los ordenadores pueda tener en la experiencia matemática de los alumnos dependerá en gran parte del contexto y de las condiciones de su utilización: ¿cómo se integran las experiencias con los ordenadores en el currículum escolar?, ¿qué papel juega el maestro y los otros alumnos en el aprendizaje con ordenadores?, ¿cómo se concibe el entorno informático y qué relación tiene con los otros entornos de aprendizaje?, ¿hemos considerado las posibles diferencias individuales que interactúan con las particularidades 
del medio informático?, etc. Estas preguntas son innumerables. Las respuestas pueden también ser muy variadas y pueden ayudar a matizar los resultados generales sobre el cambio cognitivo en el aprendizaje con ordenadores; en cualquier caso es indispensable formularlas haciendo explícitos los principios psicológicos y educativos que rigen cualquier experiencia educativa (De Corte, 1990; Martí, 1989; Papert, 1981; Solomon, 1987; Vitale, 1990). Sin poder entrar en detalles sobre el contexto educativo, digamos tan sólo que los postulados que guían el análisis que sigue remiten a una concepción constructivista del conocimiento en la que la actividad y la experiencia del alumno juegan un papel central en la adquisición y en el aprendizaje de nuevos conocimientos (Piaget, 1981) pero al mismo tiempo en la que el medio simbólico utilizado repercute sobre el conocimiento y sobre su representación.

\section{INTERACTIVIDAD Y COMUNICACION}

El aprendizaje de las matemáticas es un proceso constructivo, resultado de una compleja interacción entre diferentes instancias:

- la actividad del alumno (los procesos y estrategias que emplea cuando asimila conceptos matemáticos, cuando efectúa operaciones aritméticas, cuando resuelve problemas de álgebra, cuando generaliza a una nueva situación un algoritmo aprendido anteriormente, etc.) (Hiebert, 1986; Resnick y Ford, 1981; Schonfeld, 1987).

- los conocimientos y esquemas previos del alumno (las ideas y destrezas matemáticas-formales o informales como contar, noción intuitiva de número, de sucesor, conservación de la cantidad numérica, etc., que tienen los alumnos antes de aprender nuevos conocimientos matemáticos) (Baroody, 1988; Gelman y Gallistel, 1978; Piaget, 1975).

- los conceptos y reglas del mundo de las matemáticas (con su grado elevado de abstracción, formalización y descontextualización, su rigor y ausencia de ambigüedad en la aplicación de sus reglas, su notación simbólica, etc.) (Davis y Hersh, 1988, 1989; Gómez-Granell, 1989).

- un contexto social de intercambio planificado (el de enseñanzaaprendizaje) en el que estos tres elementos toman cuerpo (Coll, 1988; Forman, 1989; Schubauer-Leoni y Perret-Clermont, 1980).

Si aceptamos esta visión constructiva de la cognición y del aprendizaje de las matemáticas, no nos será difícil aceptar que cualquier medio que facilite la interacción entre la actividad del alumno y el objeto de aprendizaje y entre el alumno y otros interlocutores (maestro y compañeros) puede afectar de manera sustancial el proceso de aprendizaje.

Todos los autores interesados en la utilización educativa de los ordenadores coinciden en la idea de que el medio informático facilita la interacción entre el alumno y el objeto de aprendizaje si lo comparamos con otros medios como la escritura, las imágenes o el lenguaje escrito (Greenfield, 1985; Papert, 1981; Solomon, 1987). Esta facilitación es el resultado conjunto de dos propiedades del medio informático. Por un lado, es un medio que presenta y modifica las informaciones de manera dinámica: como en la animación de imágenes, pero contrariamente al medio escrito o a la notación matemática, en el medio informático las modificaciones sucesivas de cualquier tipo de información (imágenes, signos lingüísticos, signos matemáticos, sonidos, acciones) forman un proceso 
que se despliega en el tiempo y que es accesible al alumno por los pasos intermediarios que va dejando; el sujeto puede seguir ese proceso que se visualiza (normalmente en la pantalla o en algún dispositivo mecánico). Por otro lado, y esto es aún más importante, el alumno puede intervenir y modificar estas informaciones (normalmente a través del teclado). Esta constante interacción entre las acciones del alumno y los resultados visualizados en la pantalla favorecen un aprendizaje activo y controlado por el alumno.

\section{Ejemplo 1 Micromundos (2) LOGO para la exploración del} concepto de proporción.

Hoyles, Noss y Sutherland (1989) proponen dos dibujos y sus programas correspondientes realizados en el lenguaje LOGO a alumnos entre 12 y 14 años con el fin de ofrecerles la posibilidad de explorar de manera activa el concepto matemático de proporción. Un dibujo representa un personaje, el otro una casa; cada dibujo es la realización gráfica de un programa escrito en lenguaje LOGO (LESLI Y HOUSE) (Ver Fig. 1).

Los alumnos (que trabajan en grupos de dos ante el ordenador) pueden modificar los parámetros de cada uno de los programas, crear otros programas parecidos y constatar los cambios en la figura. Cada modificación introducida en el programa produce una modificación en la figura, lo que permite una constante rectificación y anticipación de nuevos parámetros según el objetivo perseguido por el alumno (hacer un personaje más grande, más pequeño, hacer una serie de casas cada vez más pequeñas, hacer una casa que sea el doble de otra, etc.).

FIGURA 1

Los 2 dibujos y sus programas correspondientes en lenguaje LOGO

TO LESLIE: SIZE

SHAPE 1: SIZE

LINE: SIZE * 0.4

SHAPE2: SIZE * 1.5

LINE: SIZE * 0.6

SHAPE $3:$ SIZE * 2

END

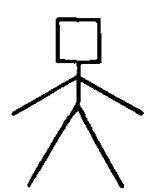

TO HOUSE

HT

FD 50

RT 60

FD 70

RT 60

FD 70

RT 60

FD 50

RT 90

FD 121

RT 90

END

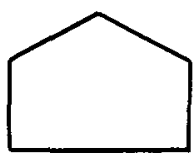


El interés matemático de estas manipulaciones e interacciones entre las acciones del alumno (que modifica el programa) y el resultado gráfico que aparece en la pantalla reside en las diferencias de los dibujos obtenidos mediante una estrategia aditiva (se añade o se sustrae una cantidad a la variable de referencia -en el programa LESLI es la variable «SIZE»—) o una estrategia multiplicativa (se multiplica el valor del parámetro por una cantidad para aumentar o disminuir proporcionalmente el valor de referencia). La primera es inadecuada (los alumnos constatan las insuficiencias en los dibujos cuando la utilizan) para realizar figuras completas y proporcionadas.

Una de las consecuencias de esta constante interacción entre la actividad del alumno y los resultados («feed-back») que va obteniendo en la pantalla es el sentimiento de control que el alumno tiene de su propio aprendizaje (es él quien modifica las informaciones). En este sentido es cierto que el aprendizaje con el medio informático aparece como un aprendizaje más autónomo: el alumno, con ayuda del profesor, diseña un proyecto con un objetivo determinado (en el ejemplo comentado es la obtención de un personaje proporcionado pero más pequeño o más grande que el personaje modelo). El sentimiento que tiene el alumno de realizar su proyecto suele ir acompañado por un grado mayor de implicación y motivación intrínseca (el proyecto es en parte suyo y lo puede ir controlando) que otros aprendizajes con medios menos interactivos (Lepper y Chabay, 1985).

Esta facilidad de interacción entre las actividades del alumno y el medio informático, junto a la fácil visualización de los resultados producidos en la pantalla suelen tener un efecto inesperado en los aprendizajes: favorecen el intercambio y la colaboración entre compañeros (Hawkins, Sheingold, Gearhart y Berger, 1982; Lepper, 1985; Webb, 1984). El trabajo con ordenadores parece pues adecuado para aprendizajes en grupo (la mayoría de las experiencias educativas con ordenadores se realizan en pequeños grupos). Esto podría originar una dinámica social conveniente para la experiencia matemática, experiencia concebida muchas veces como un doloroso itinerario solitario.

\section{INTEGRACION DE DIFERENTES MEDIOS SIMBOLICOS}

Una de las necesidades de cualquier aprendizaje que supone el dominio de un código simbólico, pero especialmente el de las matemáticas, es la de dominar la traducción de las informaciones de un medio a otro: de la acción a la notación matemática (la acción de añadir puede ser simbolizado por el signo «+»), de la formulación oral o escrita de un enunciado a su traducción matemática (pasar del enunciado de un problema a su formulación matemática), de los gráficos e imágenes a la notación matemática (pasar de la visualización de un triángulo a su definición analítica), de la notación matemática a la información gráfica (pasar de la fórmula de una función a su representación gráfica), etc. A más de ser una necesidad intrínseca de la comprensión matemática, la traducción de un código a otro es un proceso que el alumno hace con naturalidad ( $\mathrm{y}$ a veces de manera inadecuada) cuando aborda una tarea matemática: utiliza esquemas familiares de tipo figurativo, práctico o lingüístico para abordar situaciones representadas en un código matemático. Por esto, una de las dificultades en el aprendizaje de las matemáticas es el saber traducir de manera adecuada informaciones de un código a otro (Gómez-Granell, 1985; Rivière, 1990; Sastre y Moreno, 1976-77). Esta labor de traducción, además de ser un requisito para 
el aprendizaje matemático, favorece las habilidades metacognitivas del alumno: al disociar la forma simbólica del contenido representado, el alumno se da cuenta con más facilidad de que cada forma simbólica le ofrece informaciones diferentes del mismo contenido (Olson, 1974; Solomon, 1979; Dickson, 1989).

El medio informático, al ser capaz de presentar de manera simúltanea diferentes códigos simbólicos y al facilitar de manera dinámica el paso de uno a otro, constituye un medio ideal para esta actividad. Con el ordenador se pueden manipular signos lingüísticos (escritos, auditivos) y gráficos, de tal forma que se facilita la correspondencia entre ambos signos. Pensemos en la facilidad con que se puede pasar, mediante el ordenador, de una función algebráica a su representación gráfica, de una manipulación de signo (suma, resta, división) al resultado en imágenes concretas de esta manipulación, o de una notación simbólica de acciones (avanzar, retroceder, girar) a su representación gráfica.

\section{Ejemplo 2. Representación gráfica de ecuaciones}

Un programa ideado por Dugdale y Kibbey (1983) permite con facilidad visualizar el resultado gráfico de ecuaciones lineales y de segundo grado. Los alumnos escriben ecuaciones y ven al mismo tiempo el resultado gráfico. La tarea se realiza en un contexto de juego: el objetivo es escribir una ecuación (o modificar los parámetros de una ecuación) de tal forma que su representación atraviese una serie de puntos situados en la pantalla. De esta forma el alumno puede ir variando con facilidad los datos de su ecuación y establecer una correspondencia entre estas modificaciones y el resultado gráfico, hecho que puede facilitar una mejor comprensión del significado de las ecuaciones.

Este ejemplo no nos ha de conducir a la idea, errónea y peligrosa, que el medio informático es autosuficiente: cualquier ejercicio que se realice con ordenadores debería ir acompañado simultáneamente de otros ejercicios noinformáticos pertinentes. En el caso que acabamos de discutir, la contrucción gráfica por parte del alumno (con papel milimetrado, lápiz, compás y regla) de las ecuaciones escritas podría ser un buen complemento a la modificación de los parámetros de las ecuaciones y a su exploración en la pantalla del ordenador. Ambos ejercicios podrían de todas formas ser integrados (por ejemplo, haciendo dibujar la representación de una ecuación con papel y lápiz y compararlo luego con el dibujo que ofrece el ordenador).

\section{CONOCIMIENTO DECLARATIVO, CONOCIMIENTO PROCEDIMENTAL}

Una de las características del aprendizaje de las matemáticas es la constante interacción entre los aspectos declarativos (o conceptuales) del conocimiento (saber que un cuadrado es una figura cerrada que tiene cuatro lado iguales y que sus ángulos interiores son de $90^{\circ}$, saber que la operación de multiplicación $12 \times 4$ consiste en repetir cuatro veces el conjunto de 12 elementos, saber que una igualdad «a $=\mathrm{b}$ » no se altera si se resta la misma cantidad a ambos miembros, etc.) y los conocimientos de tipo procedimental (saber cómo dibujar un cuadrado, saber cómo obtener el resultado de una multiplicación, saber cómo pasar elementos de una igualdad $-\ll a-b=0 »-$ de un lado $a$ otro, etc.). Los primeros son un conjunto de significados relacionados y organizados. Los segundos son un conjunto de reglas de acción cuya aplicación conduce a cierto resultado. Ambos aspectos son siempre solidarios pero la relación entre ambos 
es difícil de establecer para el alumno. Muchas veces el alumno tiene una serie de conocimientos matemáticos pero le es difícil aplicar la regla que le permita resolver un problema (por ejemplo sabe lo que es la suma y la multiplicación pero le es difícil escoger cuál de los dos algoritmos es pertinente para resolver un problema algebráico); otras veces aprende reglas pero le es difícil aplicarlas de manera pertinente a nuevos casos (por ejemplo conoce y sabe aplicar el algoritmo de la resta llevando pero cuando el número de arriba es menor que el de abajo sigue restando el menor del mayor). En ambos casos, la dificultad reside en articular los conocimientos declarativos con los conocimientos procedimentales (Gómez-Granell, 1990). Una de las características de los sujetos expertos en relación con los sujetos novatos es precisamente una buena articulación entre ambos tipos de conocimiento (Chi, Glaser y Farr, 1988).

El medio informático se presta a que el alumno explore la vinculación de ambos tipos de conocimientos ya sea porque descarga al alumno de una carga mental excesiva haciendo de manera rutinaria ciertas operaciones, ya sea porque le exige traducir de manera procedimental sus conocimientos de tipo declarativo.

Por un lado, existe la posibilidad de que el ordenador se encargue de ejecutar una serie de reglas de manera rutinaria (por ejemplo las que permiten despejar una incógnita de una ecuación de primer grado - sumar un número a ambos lados, restar un número a ambos lados, aplicar la propiedad distributiva, etc.-) si el alumno las escoge e indica a qué parte de la expresión las quiere aplicar. En este caso el alumno no ha de buscar en su memoria estas reglas, ni ha de ejecutar los cálculos; sólo ha de escoger cuando ha de aplicarlas y evaluar entonces el resultado de su aplicación. Esto le permite explorar con más facilidad la pertinencia de aplicación de estas reglas según el tipo de expresiones y la etapa de resolución en que se encuentra (Pea, 1985).

Por otro lado, muchas de las utilizaciones de los ordenadores exigen que el alumno defina y articule una serie de procedimientos (conjunto de instrucciones básicas) para llegar a un objetivo determinado. Pensemos por ejemplo en la utilización de LOGO en su modalidad «Micromundo de la tortuga». Cuando el alumno se propone realizar un proyecto gráfico (por ejemplo dibujar una casa) ha de dar una serie de instrucciones (AVANZAR DE UNA CIERTA DIS. TANCIA, GIRAR A LA DERECHA DE CIERTO ANGULO, AVANZAR DE NUEVO DE UNA DISTANCIA MAS PEQUEÑA, etc.) que ejecuten paso a paso el dibujo de una casa. En este caso, habrá de vincular de una manera u otra los conocimientos de tipo declarativo que tiene sobre la figura que quiere dibujar (las paredes son perpendiculares, el techo está inclinado, la puerta está en medio de la fachada principal, etc.) y la definición algorítmica que le propone el lenguaje de programación.

\section{Ejemplo 3. Dibujo de un cuadrado inclinado en el Micromundo de la Tortuga (LOGO)}

Se trata de una situación que ilustra las dificultades de un niño de 11 años (Carlos) cuando ha de integrar el conocimiento declarativo con el procedimental, siendo ambos, conocimientos relativos a la figura de un cuadrado. La situación está sacada de una investigación exploratoria en la que observamos el proceso de aprendizaje del lenguaje LOGO en tres sujetos de 11-12 años (Dionnet, Martí, Vitale y Wells, 1985; Martí, 1984). 
A los 11 años, Carlos conoce sin dificultad las propiedades principales de un cuadrado (lados de igual longitud, paralelos, ángulos de $90^{\circ}$ ) y tampoco tiene dificultad en reconocer que un cuadrado que reposa sobre uno de sus vértices en vez de reposar sobre uno de sus lados, sigue siendo un cuadrado (reconocimiento que plantea dificultades a niños más pequeños como demostraron Piaget, Sinclair \& Vinh Bang, 1971, en su estudio sobre la identidad). Pero a la hora de traducir estos conocimientos estáticos, de orden declarativo, en un conocimiento procedimental (dar, en lenguaje LOGO, las instrucciones pertinentes para dibujar un cuadrado inclinado), surgen ciertas dificultades.

Carlos, escribe primero un programa para dibujar un cuadrado. Cuando la Tortuga está en su posición inicial (centro de la pantalla y posición vertical apuntando hacia arriba) el programa (llamado «CUAD») ejecuta un cuadrado. Pero cuando la orientación inicial de la Tortuga es diferente (por ejemplo está inclinada de $30^{\circ}$ hacia la izquierda) el programa realiza un cuadrado inclinado (que reposa sobre uno de sus vértices). Carlos está muy sorprendido de que su programa CUAD realice algo tan inesperado. Para entender mejor cómo se dibuja un cuadrado inclinado, intenta escribir paso a paso un nuevo programa.

\section{FIGURA 2}

Inicio del dibujo en un cuadrado «inclinado" con las instrucciones correspondientes en lenguaje LOGO

IZQUIERDA 30

ADELANTE 90

DERECHA 30

DERECHA 60

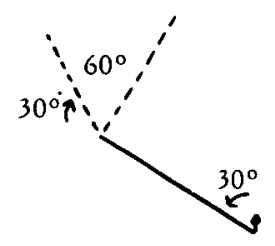

La Tortuga está en su posición inicial vertical. Carlos escribe IZQUIERDA 30 (con el objetivo de inclinar la Tortuga $30^{\circ}$ hacia la izquierda, luego ADELANTE 90 (para hacer avanzar la Tortuga de 90 pasos). Después de dibujar el primer lado se para y reflexiona un largo rato pues no sabe de cuántos grados ha de girar la Tortuga; hace con el gesto el recorrido que ha de realizar la Tortuga para dibujar el cuadrado y propone girar DERECHA 30 (girar de $30^{\circ}$ la Tortuga) justificando su elección aludiendo al giro de $30^{\circ}$ izquierda que ha realizado al empezar el dibujo. Se sorprende al ver que tras el giro de $30^{\circ}$ derecha, la Tortuga no tiene la buena inclinación (ángulo recto). Se da cuenta que tiene aún que girar más hacia la derecha y propone (correctamente) DERECHA 60 (el ángulo resultante es ahora de $90^{\circ}$ ). Dibuja el segundo lado del cuadrado, y satisfecho exclama: «Ah, se tenía que hacer un ángulo recto».

Este ejemplo muestra la dificultad de Carlos para construir paso a paso un cuadrado inclinado, partiendo de una orientación de la Tortuga inusual. Es como si este cambio de orientación de la figura repercutiese sobre las propiedades mismas del cuadrado: es entonces menos evidente que el ángulo tenga que seguir siendo de $90^{\circ}$, invariante que parecía totalmente comprendido en una aproximación declarativa del cuadrado. El ejemplo nos muestra el interés de poder pasar fácilmente de una definición y comprensión declarativa de un concepto a una definición y ejecución procedimental. 


\section{SITUACION DE RESOLUCION DE PROBLEMAS}

El hecho de conceder a la actividad del alumno un papel esencial en la elaboración de su conocimiento matemático exige que las situaciones educativas sean concebidas de tal forma que favorezcan esta exploración del mundo matemático y esta elaboración activa de los conocimientos y destrezas matemáticas. Las situaciones de resolución de problemas, frecuentemente utilizadas en la enseñanza de las matemáticas, parecen particularmente adecuadas para solicitar esta actividad. Son situaciones en las que el profesor plantea un problema al alumno dándole al mismo tiempo los elementos necesarios para su resolución. El alumno persigue pues una meta (encontrar la solución del problema) y ha de utilizar los medios adecuados para llegar a ella: ha de hacerse una representación de la meta (que muchas veces o coincide con la del profesor) y luego escoger de manera pertinente sus conocimientos y sus destrezas para aplicarlos de manera adecuada a la situación en cuestión. Vemos pues que una de las ventajas de las situaciones de resolución de problemas (comparadas a otras en las que los alumnos no persiguen un objetivo determinado de manera intrínseca por el problema) es que exigen una articulación de los conocimientos de tipo conceptual (¿cómo se representa el alumno el problema y la meta que tiene que alcanzar?, ¿con qué otro problema lo compara?, ¿qué relaciones establece entre las diferentes partes del problema?, etc.) con los procedimientos y estrategias de ejecución (¿qué submetas me propongo antes de llegar a la solución?, ¿qué reglas he de aplicar?, ¿con qué orden?, ¿cómo puedo saber que me equivoco?, etc.). Esta articulación, como hemos indicado anteriormente, es esencial para la experiencia matemática.

El hecho de que la experiencia matemática, en la medida de lo posible, se realice en situaciones de resolución de problemas puede tener otra ventaja indirecta, de orden motivacional: el alumno puede hacer suyo con más facilidad el deseo de resolver el problema (para poder superar así los obstáculos que le separan de la meta) con lo que trabajará emocionalmente más implicado y con ganas de obtener la satisfacción que conlleva llegar a la meta (3).

El medio informático se presta a la creación de situaciones de resolución de problemas. Por un lado, el medio informático, al visualizar el resultado de las manipulaciones ejercidas por el alumno (pensemos por ejemplo en los resultados gráficos obtenidos al modificar un program escrito en LOGO tal y como han sido expuestos en los ejemplos anteriores) ofrece la posibilidad de controlar si un objetivo ha sido o no alcanzado. En este sentido, el alumno puede evaluar la diferencia entre su proyecto y el resultado obtenido con mayor facilidad que en otras situaciones, lo que favorece las actividades de regulación de la propia conducta encaminadas a acercarse a un fin determinado. Por otro lado, el ordenador se presta a la simulación misma del proceso de resolución de algunos problemas y ofrece de esta manera al alumno la posibilidad de intervenir en algunas de las etapas de esta resolución. Esto permite que, ante problemas nuevos que exigen una carga atencional fuerte y que exigen una gran variedad de operaciones, el alumno pueda concentrarse en los aspectos más interesantes del proceso.

\section{Ejemplo 4. Resolución de ecuaciones algebráicas}

«AlgebraLand» constituye un Micromundo informático cuyo objetivo es el de favorecer la experiencia de los alumnos con el álgebra mediante una activa 
participación en la resolución de problemas algebráicos (Pea, 1985). El punto de partida es una ecuación algebráica que ha de ser resuelta, por ejemplo $4(2+N)$ $=20$. A la derecha de la pantalla, aparece una lista de los operadores que el alumno puede escoger para aplicarlos a toda o parte de la expresión (añadir a ambos lados de la ecuación, sustraer a ambos lados de la ecuación, multiplicar, dividir, aplicar la propiedad distributiva, combinar términos, etc.). El alumno debe pues seleccionar el operador y seleccionar dónde lo aplica. Al ejecutarlo, genera una nueva expresión. Al mismo tiempo que va resolviendo la ecuación van apareciendo todas las expresiones intermediarias así como los operadores escogidos (Fig. 3). Si el alumno explora diferentes procedimientos (porque ha aplicado de manera incorrecta los operadores o porque quiere comparar caminos diversos), la pantalla recoge, en otra ventana, cada uno de ellos y los visualiza en forma de diagrama arborescente.

Lo interesante de esta situación reside en la posibilidad que tiene el alumno de concentrarse en una de las partes de la tarea (escoger el operador así como su campo de aplicación) sin tener que calcular la aplicación aritmética de

Figura 3

Un ejemplo de dos resoluciones de una misma ecuación algebráica utilizando el Micromundo «algebraland».

(0) $4(2+N)=20$

(1) $\frac{4(2+N)}{4}=\frac{20}{4}$

(2) $2+N=5$

(3) $2+N-2=5-2$

(4) $N=3$

(5) $\mathrm{N}=3$

(0) $4(2+N)=20$

(1) $8+4 \mathrm{~N}=20$

(2) $8+4 \mathrm{~N}-8=20-8$

(3) $4 \mathrm{~N}=12$

(4) $\frac{4 \mathrm{~N}}{4}=\frac{12}{4}$

(5) $\mathrm{N}=3$

(6) $N=3$
(PROBLEMA)

(DIVIDIR)

(SIMPLIFICAR)

(SUSTRAER)

(SIMPLIFICAR)

(RESUELTO)

(PROBLEMA)

(DISTRIBUIR)

(SUSTRAER)

(SIMPLIFICAR)

(DIVIDIR)

(SIMPLIFICAR)

(RESUELTO) 
dichos operadores (el ordenador calcula) y sin tener que evocar a cada momento el conjunto de operadores posibles (pues los debe tan sólo seleccionar). Esto deja mayor libertad mental para ensayar soluciones y entender su repercusión en la solución de la ecuación. Esto no significa sin embargo que el trabajo que en este caso realiza el ordenador (cálculo aritmético y listado de los operadores) no tenga que ser trabajado con el alumno en otra ocasión. El programa admite también cierta movilidad para retroceder a un paso anterior, rectificar un operador o visualizar, como ya se ha dicho, las diferentes secuencias ensayadas.

Este panorama de la utilización del ordenador para el aprendizaje de las matemáticas puede aparecer al lector peligrosamente optimista. Lo es si se espera ingenuamente que el ordenador nos dé, por el mero hecho de utilizarlo en un contexto escolar, resultados altamente satisfactorios. Aunque en estas páginas nos hemos limitado a analizar prioritariamente el medio informático sin ocuparnos de las condiciones de su utilización, ya hemos señalado anteriormente la ilusión tecnocéntrica que consiste en esperar repercusiones positivas del ordenador sin considerar el contexto de su utilización y sin considerar el largo camino que hay que hacer para lograr una utilización provechosa de la informática en la escuela. Como acabamos de ver, el medio informático posee ciertas características que lo convierten potencialmente en un medio simbólico de gran interés para el aprendizaje en general y para el aprendizaje de las matemáticas en particular: es un medio interactivo y dinámico, que solicita el intercambio entre alumnos, que facilita la interación de diferentes tipos de códigos, que se presta para poner en relación aspectos declarativos y aspectos procedimentales y que es idóneo para un planteamiento del aprendizaje a través de situaciones de resolución de problemas. Estas ventajas no son exclusivas del medio informático. Se pueden imaginar situaciones didácticas no-informáticas que sean interactivas y dinámicas, que soliciten el intercambio entre alumnos, que faciliten la integración entre diferentes códigos, que pongan en relación los aspectos procedimentales y declarativos y que sean situaciones de resolución de problemas. Lo que ocurre es que el medio informático se presta mejor que otros medios a realizar estos objetivos. Y esta facilitación puede provocar cambios cualitativos importantes en la manera de aprender como hemos señalado a lo largo de estas páginas (4).

Esto no nos ha de conducir a desestimar los otros medios simbólicos. Ninguno es perfecto, cada uno aporta su manera de aprenhender la realidad, y seguramente el alumno llega aun conocimiento más consolidado si realiza un aprendizaje que combina e integra diferentes medios. Sería ilusorio pensar que la utilización del ordenador puede ser exclusiva en el aprendizaje en general y en el de las matemáticas en particular. Por ejemplo, el gran ausente de la interacción alumno-ordenador es el cuerpo del alumno y su implicación directa en el conocimiento. Es bueno que el alumno manipule objetos empleando todos su sentidos, se mueva, se sitúe y oriente en el espacio, mida transportando una unidad, transforme materiales diversos, etc., y que sólo manipule símbolos informáticos mirando una pantalla, sobre todo en las primeras fases de su experiencia matemática. Con el medio informático, el alumno manipula signos que representan la realidad pero raras veces manipula directamente esta realidad (visual, sonora; táctil, kinestésica). Olvidar esta limitación de la interacción con ordenadores sería no hacer caso de uno de los postulados básicos del constructivismo: el conocimiento tiene sus raíces en la acción sensoriomotora. 


\section{4}

Notas

1 El concepto de «medio» es muy amplio. Podríamos definirlo como cualquier objeto (material, simbólico) que ayude a alcanzar un fin determinado. En este sentido, la cognición humana (memoria, inteligencia, resolución de problemas) está íntimamente asociada con la utilización de medios de diferente complejidad (un objeto material - papel, lápiz, pizarrauna acción, un gesto, un índice, un signo) (Cassirer, 1944; Olson, 1976; Vygotsky, 1979).

Los más estudiados y los que mayor impacto tienen en la cognición humana son los medios simbólicos. Los medios suelen, en este caso, transcender las limitaciones de la mente (por ejemplo la carga atencional o la capacidad de memoria a corto plazo) y modifican profundamente la cognición y el conocimiento de la realidad (Cole \& Griffin, 1980).

2 En el ámbito informático, y desde que Papert (1981) utilizase este término, «micromundo» se refiere a un "objeto" informático ideado para ofrecer al alumno un terreno de experiencia y exploración en un ámbito determinado. Los micromundos pueden variar en complejidad y generalidad. El micromundo de la Tortuga está constituido, por ejemplo, por un dispositivo luminoso que puede trasladarse y dejar trazos en la pantalla cuando se comunican al ordenador una serie de instrucciones del lenguaje de programación LOGO; la Dinamotortuga es otro micromundo en el que el dispositivo luminoso puede ser accionado por instrucciones simples pero en este caso el movimiento de la tor tuga viene regido por las leyes de Newton; se pueden concebir pues múltiples micromundos que faciliten la exploración de los alumnos de determinadas leyes, conceptos o reglas matemáticas, físicas, musicales, etc.

3 Esto no quiere decir en absoluto que el planteamiento de cualquier problema vaya a apasionar automáticamente a todos los alumnos. La motivación y el grado de interés son elementos demasiado complejos e interactúan con demasiados otros aspectos (individuales, pero también interindividuales, ligados tanto al diseño de las situaciones educativas como al papel del profesor y a su relación con el alumno) (Rivière, 1990) como para ponerlos en relación sencilla con la situación de resolución de problemas. Creemos sin embargo que la situación de resolución de problemas debidamente diseñada puede favorecer el grado de implicación e interés del alumno por el hecho de que muestra con más claridad que otras situaciones el objetivo a alcanzar y por esto contribuye a dar sentido a las actividades que realiza el alumno.

4 Es difícil demostrar con rigor que un nuevo medio introduce cambios cualitativos en la manera de conocer y aprender y que no se trata de una diferencia tan sólo cuantitativa. Para convencerse de tal cambio cualitativo pensemos en la diferencia entre el conocimiento y su transmisión antes y después del descubrimiento de la escritura, antes y después del descubrimiento de la imprenta, antes y después del descubrimiento de la informática. O pensemos en lo que aporta la posibilidad de notación decimal en la experiencia numérica si la comparamos a una notación gestual o a la notación romana. En todos estos ejemplos aparece una transformación profunda del conocimiento y de su margen de aplicación debido a un cambio en el medio utilizado.

\section{Referencias}

BARoODY A. J. (1988).El pensamiento matemático de los niños. Madrid: MEC-Visor.

CAssirer, E. (1944). An essay on man: An introduction to a philosopby of buman culture. New Haven, CT: Yale University Press.

Chi, M.T.H., GLASER, R y FARR, M. (Eds,) (1988). The nature of expertise. Hillsdale, N. J.: Lawrence Erlbaum.

Cole, M. y Griffin, P. (1980). Cultural amplifiers reconsidered. En D. R. Olson (Ed.), The social foundations of language and thougth: Essays in bonor of Jerome Bruner (pp. 343-364). New York: Norton.

Colt, C. (1988). Significado y sentido en el aprendizaje escolar. Reflexiones en torno al concepto de aprendizaje significativo. Infancia y Aprendizaje, 41, 131-142

Davis, P. J. y Hersh, R.(1988). Experiencia matemática. Madrid: MEC-Labor.

Davis, P. J. y Hersi, R. (1989). El sueño de Descartes. Madrid: MEC-Labor.

DE Corte, E. (1990). Aprender en la escuela con las nuevas tecnologías de la información: Perspectivas desde la psicología del aprendizaje y de la instrucción. Comunicación, Lenguaje y Educación, 6, 93-113.

Dickson, W. P. (1989). ¿Software para hacer pensar? Sobre la yuxtaposición de los sistemas simbólicos. Comunicación, Lenguaje y Educación, 3-4, 23-38. 
Dionnet. S., Marti. E., Vitale, B y Wells, A. (1985). Représention et contrôle global-local du mouvement chez l'enfant dans la programmation LOGO. Revue Française de Pédagogie, 72, 13-23.

Dugdale, S. y Kibbey. D. (1983). Graphing equations. Iowa City: Conduit.

Forman, E. (1989). The role of peer interaction in the social construction of mathematical knowledge. Intemational Joumal of Educational Research, 13, (1), 55-70

Gelman, R. y Gallistel, C.R. (1978). The child's understanding of number. Cambridge., Mass: Harvard University Press.

Gomez-Granell, C. (1985). La representación de la multiplicación aritmética; una experiencia de aprendizaje. Infancia y Aprendizaje, 31, 229-239.

Gomez.Granell, C. (1989). La adquisición del lenguaje matemático: Un difícil equilibrio entre el rigor y el significado. Comunicación, Lenguaje y Educación, 3-4, 5-15.

Gomez.Granell, C. (1990). Estrategias de aprendizaje en la psicopedagogía de las matemáticas. En C. Monereo (Ed.), Enseñar a aprender y a pensar en la escuela. Monografía de Infancia y Aprendizaje, 31-46.

GreEnfield, P. M. (1985). El niño y los medios de comunicación. Los efectos de la televisión, videojuegos y ordenadores. Madrid: Morata.

Hawinins, J., Sheingold, K., Gearhart, M. y Berger, C. (1982). Microcomputers in schools: Impact on the social life of elementary classroom. Joumal of Applied Developmental Psychology, 3, 361-373.

HienerT. J. (1986). Conceptal and procedural knowledge: The case of mathematics. Hillsdale, NJ: Lawrence Erlbaum.

Hoyles, C., Noss, R. y Sutherland, R. (1989). Joumal of Computer Assited Leaming, 5, 208-222

Lepper, M. R. (1985). Microcomputers in education: Motivational and social issues. American Psychologist, 40, 1-18.

Lepper, M. R. y Chabay, R. W. (1985). Intrinsic motivation and instruction: Conflicting views on the role of motivational processes in computer-based education. Educational Psychologist, 20, 217-230.

MARTI, E. (1984). El ordenador como metáfora: las posibilidades educativas de LOGO. Infancia y Aprendizaje, 26, 47-64.

MArTi. E. (1989). Cuestiones y retos de la psicologia (Sección 5, Psicología, educación y nuevas tecnologías, pp. 147-181). Barcelona: Laia, Cuadernos de Pedagogía.

MARTI, E. (1990). Resolución de problemas en la interacción con el ordenador. En C. Monereo (Ed.), Enseñar a aprender y a pensar en la escuela. Monografía de Infancia y Aprendizaie, 47-66.

OLson. D. R. (Ed.). (1974). Media ans symbols: The form of expression, communication and education (73 rd Yearbook of the National Society for the Study of Education). Chicago: University of Chicago Press.

Olson, D. R. (1976). Culture, technology and intellect. En L. B. Resnick (Ed.), The nature of intelligence (pp. 189-202). Hillsdale, NJ: Lawrence Erlbaum.

OLSON, D. R. (1985). Computers as tools of the intellect. Educational researcher, 14, 5-8.

PAPert, S. (1981). Desafio a la mente. Computadoras y educación. Buenos Aires: Galápago.

$\mathrm{PEA}, \mathrm{R}$. D. (1985). Beyond amplification: Using the computer to reorganize mental functioning. Educational Psychologist, 20 (4), 167-182.

PIAGET. J. (1975). Introducción a la epistemología genética, 1: El pensamiento matemático. Buenos Aires: Paidós.

Praget, J. (1981). La teoría de Piaget. Infancia y Aprendizaje, Monografía 2 (Piaget), 13-54.

Piaget, J., Sinclair. H, y VInH.BAng (1971). Epistemologia y psicología de la identidad. Buenos Aires: Paidós.

Resnick, L. B. (1989). Developing mathematical knowledge. American Psychologist, 44 (2), 162-169.

Resnick. L B. y Ford, E. W. (1981). The psychology of matbematics for instruction. Hillsdale, N.J: Lawrence Erlbaum.

Riviere, A. (1990). Problemas y dificultades en el aprendizaje de las matemáticas: una perspectiva cognitiva. En A. Marchesi, C. Coll y J. Palacios (Eds.), Desarrollo psicológico y educación, III. Necesidades educativas especiales y aprendizaje escolar (pp. 155-182). Madrid: Alianza Psicología.

SALOMON, G. (1979). Interaction of media, cognition and learning. San Francisco: Jossey-Bass.

Sastre, G y Moreno, M.(1976-77). Représentation graphique de la quantité. Bulletin de Psychologie, $327, \mathrm{XXX}, 346-355$.

Schubauer.Leoni, M. L. y Perret.Clermont, A.N. (1980). Interactions sociales et représentations symboliques dans le cadre de problèmes audditifs. Recherches en didactique des mathématiques, 1(3), $297-350$.

Schonfeld. A. H. (1987). Cognitive science and mathematics education. Hillsdale, NJ: Lawrence Erlbaum.

Solomon, C. (1987). Entomos de aprendizaje con ordenadores. Una reflexión sobre las teorías del aprendizaje y la educación. Barcelona: Paidós.

Vitale. B. (1990). L'intégration de l'informatique à la pratique pédagogique, I: Considérations générales pour una approcbe transdisciplinaire. Genève: Departement de l'instruction Publique, Centre de Recherches Psicopédagogiques.

VyGotsky, L.S. (1979). El desarrollo de los procesos psicológicos superiores. Barcelona: Crítica.

WEBR, N. M. (1984). Micromputer learning in small groups: Cognitive requirements and group processes. Joumal of Educational Psychology, 76, 1076-1088. 


\section{Aprender matemáticas con ordenadores.} Eduardo Martí

CL\&E,1991, 11-12, pp. 63-76

Resumen: El uso de ordenadores para aprender matemáticas pude significar un cambio cualitativo en la manera de aprender y puede ofrecer al alumno nuevas posibilidades de actuación en su experiencia matemática. Este cambio en el proceso de aprendizaje es analizado a través de cuatro dimensiones: 1) interactividad y comunicación, 2) integración de diferentes medios simbólicos, 3) articulación del conocimiento declarativo y del conocimiento procedimental y 4) situación de resolución de problemas. Cada dimensión es ilustrada con un ejemplo que muestra cómo se puede utilizar el medio informático para trabajar diferentes conceptos matemáticos.

Datos sobre el autor: Eduardo Matí trabaja en el Departamento de Psicología Evolutiva y de la Educación, en la Facultad de Psicología de la Universidad de Barcelona.

Dirección: Universidad de Barcelona. Facultad de Psicología. Departamento de Psicología Evolutiva y de la Educación. C/ Adolf Florensa s/n. 08028 Barcelona.

(C) De todos los artículos. Deberá solicitarse por escrito autorización de CL\&E y de los autores para el uso en forma de facsímil, fotocopia o cualquier otro medio de reproducción impresa. CL\&E se reserva el derecho de interponer las acciones legales necesarias en aquellos casos en que se contravenga la ley de derechos de autor. 